\title{
Toward the quantitative the interpretation of hole-free phase plate images in a transmission electron microscope
}

Harada, Ken; Malac, Marek; Hayashida, Misa; Niitsu, Koudai; Shimada, Keiko; Homeniuk, Darren; Beleggia, Marco

\section{Published in:}

Ultramicroscopy

Link to article, DOI:

10.1016/j.ultramic.2019.112875

Publication date:

2020

Document Version

Peer reviewed version

Link back to DTU Orbit

Citation (APA):

Harada, K., Malac, M., Hayashida, M., Niitsu, K., Shimada, K., Homeniuk, D., \& Beleggia, M. (2020). Toward the quantitative the interpretation of hole-free phase plate images in a transmission electron microscope.

Ultramicroscopy, 209, [112875]. https://doi.org/10.1016/j.ultramic.2019.112875

\section{General rights}

Copyright and moral rights for the publications made accessible in the public portal are retained by the authors and/or other copyright owners and it is a condition of accessing publications that users recognise and abide by the legal requirements associated with these rights.

- Users may download and print one copy of any publication from the public portal for the purpose of private study or research.

- You may not further distribute the material or use it for any profit-making activity or commercial gain

- You may freely distribute the URL identifying the publication in the public portal 


\section{Journal Pre-proof}

Toward the Quantitative the Interpretation of Hole-Free Phase Plate Images in a Transmission Electron Microscope.

Ken Harada, Marek Malac, Misa Hayashida, Koudai Niitsu, Keiko Shimada, Darren Homeniuk, Marco Beleggia

PII: S0304-3991(19)30165-2

DOI: https://doi.org/10.1016/j.ultramic.2019.112875

Reference: ULTRAM 112875

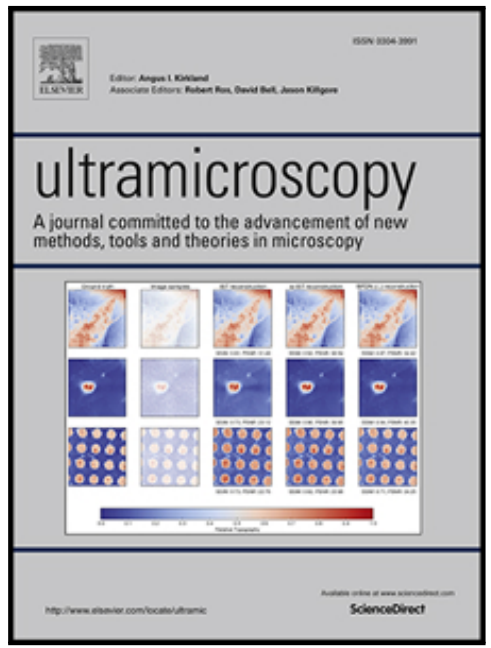

To appear in:

Ultramicroscopy

Received date:

12 May 2019

Revised date: 15 October 2019

Accepted date: 26 October 2019

Please cite this article as: Ken Harada, Marek Malac, Misa Hayashida, Koudai Niitsu , Keiko Shimada, Darren Homeniuk, Marco Beleggia, Toward the Quantitative the Interpretation of Hole-Free Phase Plate Images in a Transmission Electron Microscope., Ultramicroscopy (2019), doi: https://doi.org/10.1016/j.ultramic.2019.112875

This is a PDF file of an article that has undergone enhancements after acceptance, such as the addition of a cover page and metadata, and formatting for readability, but it is not yet the definitive version of record. This version will undergo additional copyediting, typesetting and review before it is published in its final form, but we are providing this version to give early visibility of the article. Please note that, during the production process, errors may be discovered which could affect the content, and all legal disclaimers that apply to the journal pertain.

(C) 2019 Published by Elsevier B.V. 


\section{Highlights}

- Established a quantitative link between the charge state of a hole-free phase plate and the resulting image contrast

- Used a sinusoidal diffraction grating as a phase object to separate the diffracted beams in the focal plane and isolate the resulting charge patches

- Measured the persisting phase shifts on the hole-free phase plate after irradiation with off-axis electron holography 


\title{
Toward the Quantitative the Interpretation of Hole-Free Phase Plate Images in a Transmission Electron Microscope.
}

\author{
Ken Harada ${ }^{1}$, Marek Malac ${ }^{2,3, *}$, Misa Hayashida ${ }^{2}$, Koudai Niitsu ${ }^{4}$, Keiko Shimada ${ }^{1}$, Darren
} Homeniuk $^{2}$, Marco Beleggia ${ }^{5}$

${ }^{1}$ RIKEN, Center for Emergent Matter Science (CEMS), Wako 351-0198, Japan.

${ }^{2}$ NRC-NANO, 11421 Saskatechewan Drive, Edmonton, T6G 2M9, Canada.

${ }^{3}$ Dept. of Physics, University of Alberta, Edmonton, 26G 2E1, Canada.

${ }^{4}$ Department of Materials Science and Engineering, Kyoto University, Sakyo-ku, Kyoto 606-8501, Japan.

${ }^{5}$ DTU Nanolab, Technical University of Denmark, 2800 Kgs. Lyngby, Denmark.

\section{Keywords}

Hole-free phase plate (HFPP), Volta phase plate, TEM phase contrast, cryo-TEM, electron holography, diffraction grating, electron diffraction, sample charging, radiation damage.

\section{Abstract}

We present progress toward the quantitative interpretation of phase contrast images obtained using a hole-free phase plate (HFPP) in a transmission electron microscope (TEM). We consider a sinusoidal phase grating test object composed of $\sim 5 \mathrm{~nm}$ deep groves in a $\sim 13$ $\mathrm{nm}$ thick amorphous silicon membrane. The periodic grating splits the beam current into direct beam and diffracted side beams in the focal plane of the imaging lens, where the 
HFPP is located. The physical separation between the beams allows for a detailed study of the HFPP phase shift evolution and its effect on image contrast. The residual phase shift of the electron beam footprint on the phase plate was measured by electron holography and used as input to image simulations that were compared to experimental data. Our results confirm that phase contrast is established by the phase difference between the direct and side beams, which we can estimate by fitting the image contrast evolution in time with an analytical formula describing the image intensity of a sinusoidal strong phase object. We also observed contrast reversal and frequency doubling of the grating image with time, which we interpret as the phase contrast arising from the interference between side beams becoming dominant. Another observation is the lateral displacement of the image fringes, which can be accounted for by a phase difference between the side beams.

\section{Introduction}

$\circ$ The hole free phase plate (HFPP) concept and its various implementations [1-4] have been successfully used for phase contrast enhancement in both biological and materials sciences [5-9]. The first quantitative interpretation of HFPP contrast has been demonstrated by Kotani et al. [8] for a weak phase object (WPO) where a linear relationship exists between image contrast and object phase shift. Here, we provide further insights into the interpretation of HFPP images beyond the linear regime and measure the time evolution of the HFPP phase shifts. We show that the image contrast depends on the phase difference between direct and diffracted beams, which may be significantly smaller than the phase shift of the direct and diffracted beams themselves. This point may not have been clear in the earlier reports $[1,2]$.

- To gain insight into the origin of phase contrast when a thin TEM sample is imaged with a HFPP, we fabricated a test object with a Focused Ion Beam in the form of a sinusoidal diffraction grating in a $\sim 13 \mathrm{~nm}$ thick amorphous silicon $(a \mathrm{Si})$ membrane. The grating positioned in the object plane splits the electron beam into well separated direct and diffracted beams [10]. We compared the image contrast evolution with time to image simulations without resorting to the WPO approximation. To measure the residual phase shift on the HFPP upon removing the illumination we used electron holography and found a negative value of the net phase shift [11]. Our results contribute to the understanding of HFPP phase shift evolution and its effect on the observed image contrast. The microscopic mechanisms responsible for sample charging have been subject to several recent papers [3, 4, 12-15]. At the current state of knowledge, the functionality of a HFPP originates from at least three mechanisms, often acting simultaneously: contamination build up [3], positive charging due to secondary electron emission [1] and work function alteration due to removal of surface contaminants $[2,4]$. Their relative importance depends on parameters discussed in [1-4 and 12-15]. The concept of HFPP imaging and a step by step guide to the practical operation of the phase 
plate has been described in detail in [1].

$\circ$ A periodic grating separates the direct and diffracted beams at the phase plate plane.

- The core concept in our experiments is that a periodic sample with only one strong frequency present in its Fourier spectrum splits the incident beam into three discrete and well separated beams in the back focal plane (BFP) of the imaging lens where the HFPP is located. The precise knowledge of the beam current profile and their location at the HFPP together with the evolution of the contrast provides detailed insight into the relationship between image contrast and HFPP phase shift. The known periodicity of the grating $2 L=100 \mathrm{~nm}$, as measured in a scanning electron microscope and bright field TEM (BFTEM), together with Lorentz images and electron holography of the beam footprint at HFPP allows us to calibrate the experimental parameters such as camera length $(C L=38.6 \mathrm{~mm})$ at the HFPP plane and the lateral dimensions of beam footprint on the HFPP film and phase shift at the HFPP after the experiment.

Note that in this experiment the diffraction plane is not the back focal plane of the objective lens, but a plane coinciding with the standard sample plane of the microscope while the grating is placed above the standard objective lens of the Hitachi HF-3300 S, see Figure 1.

Below we discuss the general concepts underlying the experimental setup and provide the mathematical treatment necessary to interpret the experimental results.

\section{General considerations}

A phase grating with thickness modulation $\Delta t$ and periodicity $2 L=100 \mathrm{~nm}$ adds a sinusoidal phase modulation to an incoming planar electron wavefront (Figure 1), so that the exit wave is

$\psi_{o b j}(x)=e^{i \varphi \sin \left(\frac{\pi x}{L}\right)}$

with $\varphi=1 / 2 C_{E} \Delta t V_{0}$, where $C_{E}=6.53 \mathrm{~V}^{-1} \mu \mathrm{m}^{-1}$ for $300 \mathrm{keV}$ electrons, and $V_{0}$ is the mean inner potential of the material chosen for the grating ( $V_{0}=14 \mathrm{~V}$ for $a \mathrm{Si}$ [16]). For each nm of thickness modulation in $a \mathrm{Si}$ we expect $2 \varphi=91 \mathrm{mrad}$. Grooves as deep as the entire $13 \mathrm{~nm}$ aSi film thickness would result in $2 \varphi \sim 1.18 \mathrm{rad}$ phase shift. A sample-induced phase can exceed $\varphi \sim 1 \mathrm{rad}$ as a result of Ga 
implantation and Si redeposition during the FIB fabrication of the sinusoidal grating. Qualitative insights in the contrast evolution can be obtained in the WPO approximation, but strong phase object (SPO) simulations are necessary to achieve a quantitative description of the observed contrast evolution [11].

The periodic wavefront can be decomposed into a Fourier series, representing the discrete set of plane waves that emerge from the sample:

$e^{i \varphi \sin \left(\frac{\pi x}{L}\right)}=\sum_{n=-\infty}^{+\infty} J_{n}(\varphi) e^{\frac{i n \pi x}{L}}$

Here $J_{\mathrm{n}}(\varphi)$ are Bessel functions of the first kind. The plane waves are focused into a set of spots in the diffraction plane, separated by the spatial frequency $q_{0}=1 /(2 L)$ :

$\psi_{o b j}(q)=F T\left\{\sum_{n=-\infty}^{+\infty} J_{n}(\varphi) e^{\frac{i n \pi x}{L}}\right\}=\sum_{n=-\infty}^{+\infty} J_{n}(\varphi) \delta\left(q-n q_{0}\right)$

With a periodicity $2 L=100 \mathrm{~nm}$, corresponding to a spatial frequency $q_{0}=0.01 \mathrm{~nm}^{-1}$, the scattering angle is $\theta=\lambda q_{0}=20 \mu \mathrm{rad}$ for $300 \mathrm{keV}$ electrons. For a WPO only the first diffracted beams carry significant amplitude, since when $\varphi \rightarrow 0, J_{\mathrm{n}}(\varphi) \rightarrow \varphi^{\mathrm{n}}$. For a SPO, instead, we have multiple beams contributing, each with an amplitude that oscillates non-periodically according to $J_{\mathrm{n}}(\varphi)$.

As a result of the HFPP charging locally under the diffraction pattern, we assume that each diffracted beam acquires a phase shift $\phi_{\mathrm{n}}$, which changes its complex amplitude from 1 to $\exp \left(i \phi_{\mathrm{n}}\right)$ :

$\psi_{i m g}(q)=\sum_{n=-\infty}^{+\infty} e^{i \phi_{n}} J_{n}(\varphi) \delta\left(q-n q_{0}\right)$

Once the direct and side beams recombine in the image plane, the recorded image intensity becomes:

$I(x)=\left|\psi_{i m g}(x)\right|^{2}=\left|\sum_{n=-\infty}^{+\infty} J_{n}(\varphi) e^{i\left(n k_{0} x+\phi_{n}\right)}\right|^{2}$

with $k_{0}=2 \pi q_{0}=\pi / L$. Eq. 5 is general and applies to SPO's and arbitrary phase shifts at the HFPP. Here $\phi_{\mathrm{n}}$ is the HFPP-induced phase shift of the $n$-th order beam at the HFPP plane. i.e. the $\phi_{0}$ is 
phase shift of the direct beam marked DB in Figure 2b. $\phi_{1}, \phi_{-1}$ are the phase shift of the two first order diffracted beam marked $\mathrm{SB}_{1}$ and $\mathrm{SB}_{-1}$ in Figure $2 \mathrm{~b}$.

Equation (5) can be simplified for a WPO to gain intuitive insights on the contrast evolution. For a WPO $\varphi \rightarrow 0, J_{0}(\varphi) \sim 1$ and $J_{1}(\varphi) \sim J_{-1}(\varphi) \sim 1 / 2 \varphi$. Assuming that $\phi_{1}=\phi_{-1}$, i.e. that the two side beams have identical phase shift, we have:

$I_{W P O}(x)=1+2 \varphi \sin \left(\phi_{0}-\phi_{1}\right)\left(k_{0} x\right)$

Higher order beams can be neglected because $J_{\mathrm{n}}(\varphi)=O\left(\varphi^{\mathrm{n}}\right)$. Equation 6 coincides with the image intensity from a Zernike phase plate producing a $\Delta \phi=\phi_{0}-\phi_{1}$ phase shift $[17,18]$.

As will be further discussed in section 3, a phase difference between diffracted beams, i.e. when $\phi_{1} \neq \phi_{-1}$, results in the lateral displacement of image features. This can be interpreted with a generalization of Eq. 6 to the case $\phi_{-1} \neq \phi_{1}$ :

$I(x)=1+2 \varphi \sin \left[\phi_{0}-\frac{1}{2}\left(\phi_{1}+\phi_{-1}\right)\right] \sin \left[\frac{1}{2}\left(\phi_{1}-\phi_{-1}\right)+k_{0} x\right]$

Equation 7 can be understood by noting that the term $1 / 2\left(\phi_{1}+\phi_{-1}\right)$ in the bracket is the average phase shift of the two side beams, so that the equivalent Zernike phase shift is now the difference between direct beam phase shift and this mean value: $\Delta \phi=\phi_{0}-1 / 2\left(\phi_{1}+\phi_{-1}\right)$, while $1 / 2\left(\phi_{1}-\phi_{-1}\right)$ is the amount of lateral shift of the image fringe pattern. Equation 7 can be used to extract the phase shift difference both between the direct and diffracted beams and the diffracted beams themselves if the object phase shift is small, i.e. well within the WPO.

Equation 7 fails to capture a key feature of the observed image contrast: the frequency doubling that occurs when the equivalent Zernike shift crosses multiples of $\pi$. To interpret this feature, we need to keep second order terms in the Taylor expansion of Eq. 5. This leads to an extra term

$\frac{\phi^{2}}{4}\left(\cos \left[\phi_{0}-2 k_{0} x\right]+\cos \left[\phi_{0}+2 k_{0} x\right]-2 \cos \left[\phi_{1}-\phi_{-1}+2 k_{0} x\right]\right)$ 
- to be added to Eq. 7. Note that the frequency doubling appears explicitly as $2 k_{0}$, and can occur also when $\Delta \phi$ vanishes. In this case, phase contrast originates from the direct interference between side beams. We use Eqs. 7 and 8 as model function to fit the data for the purpose of extracting values for $\varphi, \phi_{0}, \phi_{1}$, and $\phi_{-1}$ at various times during the experiment, to eventually reconstruct their time evolution.

\section{$\bigcirc$ The intensities of the diffracted beams}

- In this section we discuss the beam current distribution at the HFPP and the settling behavior of the HFPP. Assuming that the beam current density in the sample plane is $I_{\mathrm{p}} / \pi \mathrm{R}_{\mathrm{i}}{ }^{2}$, where $I_{\mathrm{p}}$ is the total beam current and $R_{\mathrm{i}}$ is the radius of the illuminated patch, the total current carried by each diffracted beam will be $I_{\mathrm{p}} J_{\mathrm{n}}{ }^{2}(\varphi)$. Assuming that no apertures are present anywhere between the sample plane and the chosen diffraction plane, the continuity equation dictates that we must find the same number of electrons at any plane perpendicular to the optic axis. In particular it applies to object and focal planes and is satisfied by the property of Bessel functions:

- $\sum_{n=-\infty}^{+\infty} J_{n}^{2}(\varphi)=1$

- that holds for any $\varphi$. It is interesting to note that for an SPO with $\varphi=2.4 \mathrm{rad}$, corresponding to the first zero of the $J_{0}$ function, the direct beam is extinct if illumination does not extend outside the grating area of the sample.

\section{$\circ$ The diffraction pattern of the phase grating charging the HFPP}

- The relationship between the phase shifts $\phi_{\mathrm{n}}$, illumination characteristics (beam current, current density and energy), geometry (convergence and collection angles, distance to the electrical contacts, choice of diffraction plane, apertures, etc.) and HFPP material parameters (composition, thickness, conductivity and general electrical properties, secondary electron (SE) yield, surface state, presence or absence of adsorbates, etc.) is complex [1-4, 12-15, 19]. While we expect a connection between diffracted spot intensity and magnitude of the resulting charge distribution, the relation is most certainly non-linear. The current density at the HFPP is primarily responsible for the rate of change at which the charge distribution evolves under each spot $[3,4,12]$, but it may also affect its magnitude upon reaching a steady state. While it seems intuitive to imagine that a positive charge distribution should arise from secondary electron emission in the thin-film geometry typical of TEM [1, 14], mounting evidence indicates that negative charging can be encountered $[3,4,12]$. Furthermore, film charging often appears to proceed in an irreversible fashion, with an enormous difference between charging and discharging time constants. The latter being several orders of magnitude slower than the former 
[20] causes persistent charging of the film weeks after the beam has been turned off.

- Here we illustrate the simplest conceivable mechanism for how a charge distribution may arise under a diffraction spot: secondary electron emission coupled to Ohmic conduction from a nearby grounded electrode $[1,13,19,21,22]$. While insufficient to capture the physics of electron-matter interactions, this model provides basic concepts that could be adapted to more realistic scenarios once the many unknown parameters are determined experimentally. With this objective in mind we aim at explaining the phase shifts $\phi_{\mathrm{n}}$ and their time evolution.

- Describing the irradiated area as a finite cylindrical capacitor of height $d$ (the HFPP thickness), inner radius $R_{\mathrm{n}}$ (the radius of the charged patch under the $n$-th beam), outer radius $R_{\mathrm{c}}$ (representing a grounded electrode located at $r=R_{\mathrm{c}}$ ), and filled with a medium of permittivity $\varepsilon$ and resistivity $\rho[1]$, we can setup the continuity equation for each beam as:

- $\frac{d V_{n}}{d t}=\frac{Y I_{n}}{C_{n}}-\frac{V_{n}}{\tau}$

- where $Y$ is the secondary electron yield, $I_{\mathrm{n}}$ is the total current carried by the $n$-th diffraction spot,

- $C_{n}=\frac{2 \pi \varepsilon}{\log \left(R_{c} / R_{n}\right)}$

- is the capacitance, and $\tau=\rho \varepsilon$ is the dielectric relaxation time, playing the role of $R C$ time constant of the system. Within these simple approximations, the potential developing under a spot depends exponentially on time according to:

- $V_{n}(t)=V_{n}^{\infty}\left(1-e^{\frac{-t}{\tau}}\right)$

- where the asymptotic potential at time $t \rightarrow \infty$ is

- $\quad V_{n}^{\infty}=\frac{Y I_{n} \rho}{2 \pi} \log \left(\frac{R_{c}}{R_{n}}\right)$

- The spatial profile of the potential is, by construction, constant for $r<R_{\mathrm{n}}$, and decaying as $\log \left(R_{\mathrm{c}} / r\right)$ for $R_{\mathrm{n}}<r<R_{\mathrm{c}}[1,24]$. The tail of the potential extends all the way to the ground 
electrode, implying a substantial overlap between the electric fields associated with each spot, which makes the model untenable. Maintaining the exponential time dependence, replacing the slow logarithmic decay with another exponential, reflecting the possibility of local screening by surrounding mobile charges, while keeping the asymptotic potential as a free parameter to be experimentally determined, allows us to compute the phase shifts [1]. In fact, at any given time, the electrostatic potential extends in the vacuum above and below the charged patch of the HFPP film. The phase shift experienced by subsequent electrons is then the projection of this potential along the optical axis $[1,11]$. The small diffraction angles coupled with potentials much smaller than the accelerating voltage insure that we can consider paraxial and straight electron trajectories.

- Assuming further that the separation between the spots is larger than the extension of the phase shift resulting from this integration, we can finally estimate the time dependence of the HFPP phase shifts as:

- $\phi_{n}(t)=C_{E} V_{n}^{\infty} R_{n}\left(1-e^{\frac{-t}{\tau_{n}}}\right)$

- where we have allowed for the characteristic times to vary for each beam, since as soon as we venture beyond the Ohmic picture at constant SE yield we should expect the time constant to depend on the beam intensity due to charge carrier generation and recombination effects. When $\mathrm{V}_{\mathrm{n}}{ }^{\infty}$ is negative $[2,3]$, the phase shift tends to a negative value [11].

\section{- 2.1 Experiment}

- In this section we discuss the fabrication of the grating sample, microscope set up and summarize the experimental results.

\section{- 2.2 Periodic grating sample fabricated using a Focused Ion Beam}

- The samples were fabricated using a dual beam instrument, Hitachi NB 5000. A $40 \mathrm{keV} \mathrm{Ga}{ }^{+}$ion beam was rastered in line patterns with a $0.5 \mu$ s pixel dwell time and $0.01 \mathrm{nA}$ ion beam current. The total number of lines was 200 with a $25 \mu \mathrm{m}$ length and $100 \mathrm{~nm}$ spacing. The grating groves were fabricated in a pristine $\sim 13 \mathrm{~nm}$ aSi film on a Si frame (Norcada Inc.). A thickness map obtained from BFTEM [23], see Figure 5b, shows that the grating period was $~ 100 \mathrm{~nm}$ and the magnitude of the thickness variations was below $6 \mathrm{~nm}$, i.e. $0.55 \mathrm{rad}$ or $\sim \pi / 5.55$. The BFTEM images exhibit diffraction contrast at the crests of the fabricated grooves suggesting $\mathrm{Ga}$ implantation and Si re-depostion. 


\section{- 2.3 TEM optics to separate diffracted and direct beam.}

- Studying the direct beam (DB) and side beams (SBs) phase shifts independently requires their lateral separation. This was achieved by utilizing a custom optics of the cold-FEG Hitachi HF3300 S (Hitachi High Technologies, Naka, Japan) operated at $300 \mathrm{kV}$. The two sample stages of the instrument, with the objective lens pre-field situated between them, allows to obtain a 1.52 $\mu \mathrm{m}$ separation between the direct and diffracted beams, corresponding to a camera length $C L=38.6 \mathrm{~mm}$ (see Figure $1 \mathrm{~b}$ and c). Standard sample holders were used at both planes making it possible to adjust the temperature of the HFPP.

- The $1.52 \mu \mathrm{m}$ separation between the beams was sufficient to prevent a "cross talk" between their phase shifts. Furthermore, by adjusting the excitation of the $1^{\text {st }}$ intermediate lens we projected the diffraction pattern onto the slow scan CCD camera, thereby capturing the beam current density distribution at the HFPP plane, as shown in Figure 2b. Taking into account the relatively large periodicity of the diffraction grating and the microscope stability, both sample and HFPP drift had a negligible effect on the observations.

\section{- 2.4 Contrast evolution, beam current density profile and phase shift at the HFPP}

- Here we discuss the beam current density distribution at the HFPP plane and the grating image contrast evolution with irradiation dose. The carbon film was placed in a heating holder held at $150^{\circ} \mathrm{C}$ and utilized as a HFPP. The carbon film was kept at $150^{\circ} \mathrm{C}$ overnight in the column vacuum that was below $2 \times 10^{-8}$ torr.

- The diffraction peaks were fitted to a set of Gaussians revealing that the peak value of the $1^{\text {st }}$ order side beam $\left(\mathrm{SB}_{1}\right)$ contains $j_{1} \sim 200 \mathrm{x}$ less beam current than the direct beam $j_{0}$ and the $2^{\text {nd }}$ order side beam $\left(\mathrm{SB}_{2}\right)$ are $j_{2} \sim 7000 \mathrm{x}$ weaker than the direct beam each $[9,10]$. The off-axis electron holography measurement of the phase shift after the grating contrast evolution was collected is shown in Figure 3. The reconstructed phase image indicated that the direct beam phase shift at the HFPP was about $\phi_{0}=-0.6 \mathrm{rad}$ while the phase shift at the locations of the $1^{\text {st }}$ order diffracted beams was about -0.2 to $-0.3 \mathrm{rad}$, resulting in a net phase shift $\Delta \phi \sim-0.4$ to -0.3 rad. The sign of the net phase shift is consistent with a negative charge patch more compact than the positive screening charge, and/or with material removal by the direct beam $[3,4$, $10,11]$. The polarity was confirmed by comparing the sign of the phase shift of the charged patch to the phase shift arising of a thin carbon film, that has positive mean inner potential, relative to vacuum outside the carbon film. In agreement with $[1,3,4]$, the phase shift at the HFPP is broader, about $10 x$, than the diameter of the incident direct or diffracted beams at the HFPP. The FWHM of the phase profile in Figure 3, is about $320 \mathrm{~nm}$ as compared to the beam current diameter $\sim 28 \mathrm{~nm}$ in Figure $2 \mathrm{a}$. Note that electron holography requires irradiating the HFPP film with a spread illumination, which may affect the measurements. However, the 
charge polarity in the asymptotic state, determined after the experiment, is reliable and the measured width of the phase profile represents a lower bound of the phase shift FWHM during HFPP operation.

- Figure 4a shows an example of an image extracted from a settling series stack of images tracking the contrast evolution as a function of cumulative irradiation dose. A series of 120 images acquired over a $600 \mathrm{~s}$ in $5 \mathrm{~s}$ intervals with $1 \mathrm{~s}$ acquisition time in Figure 4 was collected at total beam current dose rate $2.8 \mathrm{e}^{-} \mathrm{nm}^{-2} \mathrm{~s}^{-1}$ at the sample plane. Each of the 120 individual images was then collected at a dose of $\sim 2.8 \mathrm{e}^{-} \mathrm{nm}^{-2}$ at the grating plane.

- A 400 pixel long profile was extracted from each of the images in Figure 4a and plotted as a function of time in Figure 4b. Intensity profiles were then used to follow the time evolution of the image contrast. The time profile shown in Figure 5a was extracted from a location of minimum intensity and maximum intensity at $t=0 \mathrm{~s}$. An exponential decay $I(t)=I_{\mathrm{A}}[1+\exp (-$ $t / \tau)]+A$ was fitted to the extracted intensity. In this formula only the characteristic time $\tau$ is of interest, which was fitted as $\tau \sim 147 \mathrm{~s}$. While the fit of the intensity decay at particular location shown in Figure 5a does not provide full insight into the evolution of the phase shift, it is a meaningful measure of the contrast rate of change. Figure 5b shows 400-pixel-wide intensity profiles extracted from BFTEM images collected with defocused imaging lens (red sinusoidallike) and close to in-focus (yellow, nearly flat line). For reference, Figure $5 \mathrm{~b}$ also shows image intensity profile extracted from Figure 4 at 600 s mark (blue, upper line). Figure 5b, yellow profile shows that there is very little amplitude contrast in the in-focus image, confirming that the sample can be treated as a pure phase object. The BFTEM profiles were acquired within a short period of time, but the HFPP profile was acquired at a different time with a different beam current leading to an offset of the overall intensity of the HFPP profile.

- Figure 4b shows that the image contrast reverses: at about $t=150$ to $200 \mathrm{~s}$, the fringe periodicity distance (abscissa in Figure 4b) is halved, i.e. their spatial frequency doubles, as compared to the $t=0 \mathrm{~s}$ and $t>250 \mathrm{~s}$. The frequency doubling indicates that the phase shift between the direct and diffracted beams is $n \pi$ with $n$ integer [10]. Taking into account that holography measures a negative phase shift, it is likely that $n=-1$ or $n=0$ at around $t=150 \mathrm{~s}$. Taking the sum of counts across the image profile of the images in the stack allows us to estimate and account for change of HFPP thickness as a result of contamination build up or hole drilling [1, 3, 4]. Any thickness change of sample and HFPP combined as well as beam current drift was less than $5 \%$ over the $600 \mathrm{~s}$ experiment duration.

\section{- 2.5 Fitting the contrast evolution as a measurement of phase shift at the phase plate}

- We now turn our attention to the quantification of HFPP phase shifts from the intensity profiles in Figure 4. The results of the fit of Eq. 5 to the measured intensity profiles from Figure $4 \mathrm{~b}$ are 
shown in Figure 6.

- The frequency of the fringes was fixed for the purpose of the fit at $k_{0}=2 \pi / 0.063 \mathrm{~nm}^{-1}$, i.e. the real space period of the fringes, $100.2 \mathrm{~nm}$. The object phase shift $\varphi$ was also fixed at $\varphi=0.2 \mathrm{rad}$, determined from the fit as a global parameter. Experimentally, $\varphi=0.2 \mathrm{rad}$, corresponds to about $4.4 \mathrm{~nm}$ from groove bottom to its crest, assuming $14 \mathrm{~V}$ mean inner potential for $\mathrm{Si}$. The phase shift evolution of the direct beam $\phi_{0}$ and the $1^{\text {st }}$ order diffracted beams on each side of the direct beam $\phi_{1}$ and $\phi_{-1}$ (see Figure 2) and the net phase shift $\Delta \phi=\phi_{0}-1 / 2\left(\phi_{1}+\phi_{-1}\right)$ are shown in Figure $6 \mathrm{a}$ and $6 \mathrm{~b}$ respectively as a function of time. The extracted phase shift of about 0.5 to $2 \mathrm{rad}$ for each of the beams in Figure 6a agrees well with the 0.3 to 0.7 rad phase shift measured by electron holography after the experiment finished, see Figure 3. (Note: magnitude of phase shift is shown in Figure 3, the phase shift in both Fig 3 and Fig 6 correspond to negative phase shift.) Using the fitted values $\Delta \phi, \phi_{0}, \phi_{1}, \phi_{-1}$ to simulate the intensity profile evolution reproduces the experimental data Figure 4 well, as shown in Figure 6c. In particular, the frequency doubling appears at $200 \mathrm{~s}$, as in the experimental data. Furthermore, the contrast reversal and lateral shift of fringes are all properly reproduced. Note that the frequency doubling feature coincides with the net phase shift crossing zero, implying that the contrast profiles around this time can be described by equation (8) applied to $\phi_{0}=1 / 2\left(\phi_{1}+\phi_{-1}\right)$.

- Figure 6a shows the phase shift of the direct beam phase shift (blue), the $\phi_{-1}$ diffracted beam (yellow) and the $\phi_{1}$ diffracted beam (red) as extracted by fitting Eq. 5 to the experimental data from Figure 4b. Not surprisingly, the fitted yalues of the phase shift of the individual beams do not appear to have direct relation to the observed contrast and may seem unphysical. For example, the $\phi_{0}$ and $\phi_{-1}$ appear to follow the same decreasing tendency with time while $\phi_{1}$ appears to trend upwards although all beams impinge the same HFPP carbon film. However, this causes no concern because the observed image contrast does not depend on the absolute phase shifts of each beams separately, but on their combinations $\phi_{0}-1 / 2\left(\phi_{1}+\phi_{-1}\right)$ and $1 / 2\left(\phi_{1}-\phi_{-1}\right)$, according to Eqs. 7 and 8 . Furthermore, the observation that the phase shift of one of the diffracted beams exceeds that of the direct beam (visible in Fig. 6), which may appear puzzling at first sight, has a simple explanation in the choice of origin of the model function used to fit the data. Choosing, for example, a cosine instead of a sine to describe the object phase shift would result in the \pm 1 side beams retarded/advanced by $1 / 2 \pi$ with respect to the direct beam, respectively. The net phase shift $\Delta \phi$ plotted in Figure 6b shows a monotonic evolution, becoming progressively more negative with time: it starts from $\sim+0.2 \mathrm{rad}$ at $t=0 \mathrm{~s}$, then crosses zero at around the $120 \mathrm{~s}$ mark (black arrow), at the onset of the fringe frequency doubling region in the image, see Figure $4 \mathrm{~b}$ and $6 \mathrm{c}$, and finally decreases down to $\sim-0.3 \mathrm{rad}$ at the end of the experiment when the electron holograms were collected. The green line with shadowed region in Fig. 6b indicates the net phase shift measured by electron holography, see Figure 3. The $\Delta \phi$ extracted by electron holography is in good agreement with the phase shift extracted from the grating image evolution.

- To interpret the evolution of the net phase shift it is reasonable to assume that the same set of microscopic mechanisms, e.g. positive charging due to secondary electron emission [1] or 
electron stimulated desorption of water [3], acts at both the direct and diffracted beam locations. At the same time, the fact that the current density at the direct beam location is much higher than at the diffracted beams, suggests that the rate of change should differ.

- The evolution of the net phase shift from positive to negative may occur in the following scenarios:

- $\phi_{0}, \phi_{1}$ and $\phi_{-1}$ are all always positive. The charging of the direct beam is faster than the diffracted beams implying that initially $\phi_{0}$, is larger than the average of $\phi_{1}$ and $\phi_{-1}$. As charging progresses, the difference between the direct and diffracted beams decreases and eventually the phase shift of diffracted beams exceeds that of the direct beam.

- Initially the direct and diffracted beam are one polarity, e.g. positive, arising from fast acting charging process such as secondary electron emission. See (left side of the Figure 6b), [1, 21, $22]$. Over time the initial process saturates and another process takes over, such as mass loss and/or decreased work function due to electron stimulated desorption of water [3,11]. Consequently, both the direct and diffracted beams may change polarity over time leading to the observed reversal.

- While other combinations may be possible, explanation ii. is plausible because the situation at the direct and diffracted beam is essentially the same. The carbon film has the same properties at the direct and diffracted beam locations, the only difference being the local beam current density. As the diameter of the area oyer which the surface species are removed increases, the phase shift also increases although the magnitude of the potential in the clean area is constant [3]. Indeed the contrast observed in the fringes continues to increase according to Eq. 5 and Eq. 8 , albeit at decreased rate [11].

\section{- Discussion and insights from computer model results}

- In this section we use numerical simulations to illustrate the conditions that lead to frequency doubling and the generic behavior of the contrast evolution. We explore a situation where the initial net phase shift is positive, $\phi_{0}{ }^{0}=\pi / 2$, see Fig $6 \mathrm{~b}$. The initial phase shift $\phi_{0}{ }^{0}$ reflects the phase difference at the time when first data set was collected. It can be set to zero if the first data is collected at time shorter than the phase constants $\tau_{\mathrm{DB}}, \tau_{-1}$ and $\tau_{1}$. The asymptotic value of the phase shift for the direct and diffracted beams is equal and it is taken to be $\phi_{0}{ }^{\infty}=\phi_{1}{ }^{\infty}=\phi_{-1}{ }^{\infty}=-$ $2 \pi$. The $-2 \pi$ value is slightly higher than expected in most experiments, ensuring that entire practical range of HFPP operation is mapped out. With eq. (14), we estimate that the surface potential corresponding to $-2 \pi$ phase shift difference is about $5 \mathrm{~V}$ for $\sim 200 \mathrm{~nm}$ radius of the charged patch. Such a size of the charged area is realistic for an incident beam radius in the order of a few tens of nanometers. This arises from the fact that both positive charging and removal of surface molecules are driven by secondary electrons, which are typically generated 
in a direction perpendicular to the incident beam and may travel a long distance from it.

Rewriting Eq. 14 in the form:

- $\phi_{n}(t)=\phi_{n}^{\infty}\left(1-e^{\frac{-t}{\tau_{n}}}\right)(15)$

- leads to

- $\Delta \phi(t)=\phi_{0}^{0}+\left[\phi_{0}^{\infty}\left(1-e^{\frac{-t}{\tau_{D B}}}\right)-\frac{1}{2}\left(\phi_{1}^{\infty}\left(1-e^{\frac{-t}{\tau_{1}}}\right)+\phi_{-1}^{\infty}\left(1-e^{\frac{-t}{\tau_{-1}}}\right)\right)\right]$.

- Figure 7a shows a 2D phase shift map with phase shift corresponding to reversals marked with black lines at $0 \pi$, and $-\pi$ phase shift. The time constant for the diffracted beams $\tau_{-1}=\tau_{1}$ was taken $213 \mathrm{x}$ slower than that of the direct beam $\tau_{\mathrm{DB}}$. I.e. the settling time constant is proportional to beam current at the beam location. Under such conditions $n=0$ contrast reversal at $t \sim 170 \mathrm{~s}$ (marked with a white circle) implies settling time constant of the direct beam $\tau_{\mathrm{DB}} \sim 600 \mathrm{~s}$. As the experimental time increases, see orange arrow, a second reversal $n=-1$ at net phase shift $-\pi$ would appear at $t \sim 800 \mathrm{~s}$, marked with a red circle. Following the second reversal is a long plateau of very slowly varying net phase shift implying correspondingly stable image contrast. While we do not know the asymptotic values of the phase shift of the direct and diffracted beams or the respective time constant, the Figure 7a illustrates an important point: Although the asymptotic phase shift is the same for the direct and diffracted beam, a stable contrast will appear over extended period of time even for widely separated direct and diffracted beams. Figure $7 \mathrm{~b}$ was plotted under the assumption that the diffracted beams settle only $20 \mathrm{x}$ slower than the direct beam. It illustrates another important point: while the settling time constants determines the spacing of the contrast reversals during the experiment, a plateau of a stable phase shift and image contrast still appears, albeit after multiple contrast reversals during the initial stabilization period. The inset in Figure $7 \mathrm{~b}$ illustrates yet another salient feature: when the experiment duration is sufficiently long ( 14 hours simulated here), the net phase shift difference will tend to the initial net phase shift $\Delta \phi_{0}{ }^{0}$ value and the contrast will decrease. Importantly, Figure 7 shows that the existence of two settling constants does not imply two different settling processes, in disagreement with the mechanisms proposed in [2]. The different settling constants for direct and side beams can be explained by the difference in beam current at the direct and side beam locations leading to a difference in settling time, but not the settling time constant or the asymptotic phase shift. However, it is possible that more than one physical process is responsible for the net settling time constant. An example of such processes can be (fast) charging due to SE emission [1], slower development of surface potential due to electron stimulated desorption [2-4], possibly combined with thickness removal.

\section{- Conclusions}

- We have used a phase grating object to demonstrate how phase contrast arises with a hole-free phase plate. Each of the diffracted beams creates a small charged patch on the film. These 
charged areas generate an electric field that adds a phase shift, specific to each beam, to subsequent electrons. We have acquired off-axis electron holograms of the footprint of the grating diffraction pattern on the HFPP and measured their residual phase shifts after the experiment ended. When the phase-shifted beams recombine in the image plane, the periodic phase grating object becomes visible in-focus. The image intensity profile for arbitrary sets of beam phase shifts can be reliably predicted with a simple analytical model based on standard image formation theory. Since the charged patches under the diffraction pattern evolve in time, a time-series of the HFPP image of the phase grating shows the contrast features also varying in time. Fitting the time-series with the analytical model provided us with a measure of the object phase as well as of the time dependency of each of the beam phase shifts. A simple Ohmic model for the potential build up upon irradiation suggests exponential trends with beam-specific asymptotic values and time constants for the phase shifts. The measured phase difference between the side bands explains the image fringe pattern shifting laterally, as observed experimentally. The net HFPP phase shift crossing zero at $n=0$ explains another observed feature: a frequency doubling of the fringe pattern around $t=200 \mathrm{~s}$, arising from the direct interference between the two sidebands giving half-periodicity image intensity.

\section{- Acknowledgments}

- This research was financially supported NRC-NANO in Edmonton, Canada and RIKEN in Hatoyama, Japan. Support of Dr. Y. Okura, Dr. S. Motoki, Dr. I. Ishikawa and Dr. Y. Konyuba of JEOL Ltd., Akishima, Japan on development of phase plate methods in a TEM has been critical for ongoing development of phase plate microscopy at NRC-NANO. The support of Hitachi High Technologies and in particular Dr. Y. Taniguchi, has been instrumental in designing the microscope hardware needed to study phase plate contrast formation. Frequent motivating and insightful discussions with Prof. Ray Egerton are gratefully acknowledged. We also wish to acknowled ge many helpful discussions with Mr. Edward Rowan, in particular on the time evolution of contrast, and on the microscopic mechanisms behind it. We acknowledge the anonymous reviewers for an extensive proof-reading of our manuscript, and for a set of very useful comments that improved its quality.

\section{- References}

- [1] M. Malac, M. Beleggia, M. Kawasaki, P. Li, R.F. Egerton, Convenient contrast enhancement by a hole-free phase plate, Ultramicroscopy, 118 (2012) 77-89.

- [2] R. Danev, B. Buijsse, M. Khoshouei, J.M. Plitzko, W. Baumeister, Volta potential phase plate for in-focus phase contrast transmission electron microscopy, Proc Natl Acad Sci U S A, 111 (2014) 15635-15640.

- [3] S. Hettler, M. Dries, P. Hermann, M. Obermair, D. Gerthsen, M. Malac, Carbon contamination in scanning transmission electron microscopy and its impact on phase-plate applications, Micron, 96 (2017) 38-47.

- [4] S. Hettler, E. Kano, M. Dries, D. Gerthsen, L. Pfaffmann, M. Bruns, M. Beleggia, M. Malac, 
Charging of carbon thin films in scanning and phase-plate transmission electron microscopy, Ultramicroscopy, 184 (2018) 252-266.

- [5] M. Hayashida, K. Cui, A.M. Najarian, R. McCreery, N. Jehanathan, C. Pawlowicz, S. Motoki, M. Kawasaki, Y. Konyuba, M. Malac, Hole free phase plate tomography for materials sciences samples, Micron, 116 (2019) 54-60.

- [6] N. Hosogi, A. Sen, H. Iijima, Comparison of Cryo TEM Images Obtained with Zernike and Hole-Free Phase Plates, Microscopy and Microanalysis, 21 (2015) 1389-1390.

- [7] S. Pollard, M. Malac, M. Beleggia, M. Kawasaki, Y. Zhu, Magnetic imaging with a Zernike-type phase plate in a transmission electron microscope, Applied Physics Letters, 102 (2013).

- [8] A. Kotani, K. Harada, M. Malac, M. Salomons, M. Hayashida, S. Mori, Observation of FeGe skyrmions by electron phase microscopy with hole-free phase plate, AIP Advances, 8 (2018).

- [9] A. Kotani, K. Harada, M. Malac, H. Nakajima, K. Kurushima, S. Mori, Magnetic textures in a hexaferrite thin film and their response to magnetic fields revealed by phase microscopy, Japanese Journal of Applied Physics, (2019).

- [10] M. Malac, M. Hayashida, K. Harada, K. Shimada, K. Niitsu, T. Rowan, M. Beleggia, Hole-Free Phase Plate Imaging of a Phase Grating, Microscopy and Microanalysis, 24 (2018) 894-895.

- [11] M. Malac, S. Hettler, M. Hayashida, M. Kawasaki, Y. Konyuba, Y. Okura, H. Iijima, I. Ishikawa, M. Beleggia, Computer simulations analysis for determining the polarity of charge generated by high energy electron irradiation of a thin film, Micron, 100 (2017) 10-22.

- [12] S. Hettler, J. Onoda, R. Wolkow, J. Pitters, M. Malac, Charging of electron beam irradiated amorphous carbon thin films at liquid nitrogen temperature, Ultramicroscopy, 196 (2019) 161 166.

- [13] W.A. Hubbard, M. Mecklenburg, H.L. Chan, B.C. Regan, STEM Imaging with BeamInduced Hole and Secondary Electron Currents, Physical Review Applied, 10 (2018).

- [14] C.J. Russo, R. Henderson, Microscopic charge fluctuations cause minimal contrast loss in cryoEM, Ultramicroscopy, 187 (2018) 56-63.

- [15] C.J. Russo, R. Henderson, Charge accumulation in electron cryomicroscopy, Ultramicroscopy, 187 (2018) 43-49.

- [16] E. Volkl, L.F. Allard, D.C. Joy, Introduction to Electron Holography, in, Springer, Boston, MA, 1998.

- [17] M. Malac, M. Beleggia, R. Egerton, Y. Zhu, Imaging of radiation-sensitive samples in transmission electron microscopes equipped with Zernike phase plates, Ultramicroscopy, 108 (2008) 126-140.

- [18] M. Beleggia, A formula for the image intensity of phase objects in Zernike mode, Ultramicroscopy, 108 (2008) 953-958.

- [19] Q.-T. Doan, A. Hamdouni, A. El Hdiy, Surface recombination velocity effects on simulated electron beam induced current collected by a nanoscale electrode, Superlattices and Microstructures, 100 (2016) 1296-1300.

- [20] J. Brink, M.B. Sherman, J. Berriman, W. Chiu, Evaluation of charging on macromolecules in electron cryomicroscopy, Ultramicroscopy, 72 (1998) 41-52.

- [21] M. Malac, M. Beleggia, T. Rowan, R. Egerton, M. Kawasaki, Y. Okura, R.A. McLeod, Electron Beam-Induced Charging and Modifications of Thin Films, Microscopy and Microanalysis, 21 (2015) 1385-1388. 
- [22] M. Malac, M. Beleggia, R. Egerton, M. Kawasaki, M. Bergen, Y. Okura, I. Ishikawa, K. Motoki, Charging of Thin Film Phase Plates under Electron Beam Irradiation, Microscopy and Microanalysis, 20 (2014) 230-231.

- [23] H.-R. Zhang, R.F. Egerton, M. Malac, Local thickness measurement through scattering contrast and electron energy-loss spectroscopy, Micron, 43 (2012) 8-15.

- [24] M. Malac, M. Bergen, R. Egerton, M. Kawasaki, M. Beleggia, H. Furukawa, M. Shimizu, Practical hole-free phase plate imaging: principles, advantages and pitfalls, Microscopy and Microanalysis, 18 (2012) 484-485. 


\section{- Figure captions}

$\cdot$

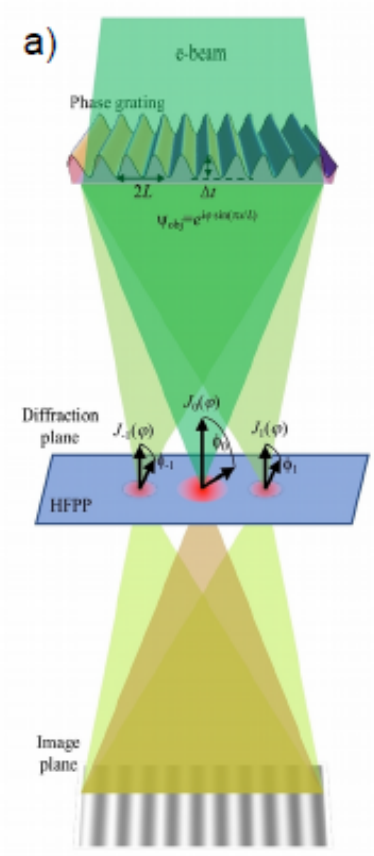

Figure 1

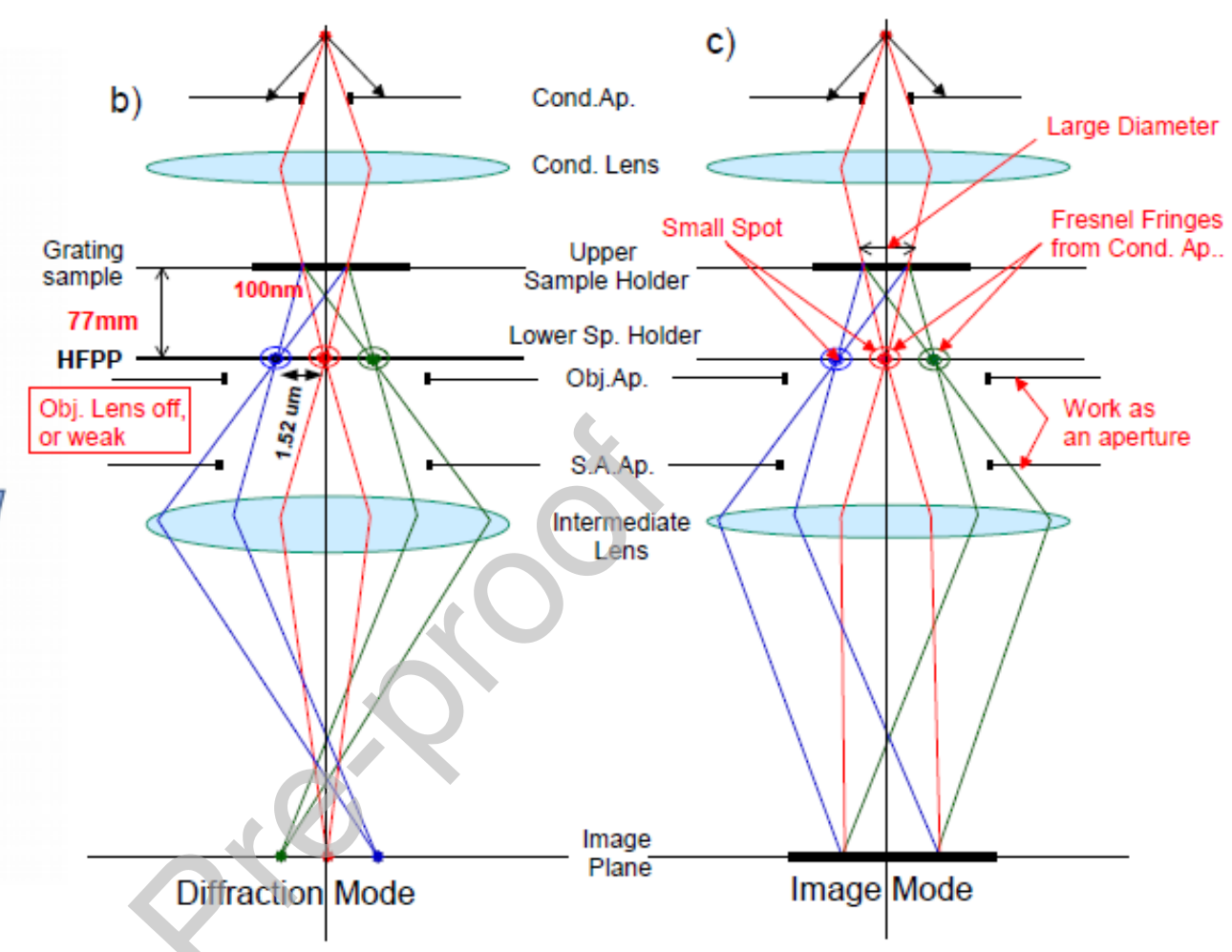

- Figure 1 (Color online) a) Conceptual outline of the experiment. A planar electron wave impinging on a sinusoidal thickness-modulated Si membrane with periodicity $2 L=100 \mathrm{~nm}$. The periodic exit wave $\psi_{\mathrm{obj}}(x)=\exp (\mathrm{i} \varphi \sin (\pi x / L))$ decomposes into a discrete set of plane waves converging to spots in the diffraction plane. Each spot, with amplitude $J_{\mathrm{n}}(\varphi)$, charges the HFPP (red dots) according to its current density, beam extension, electrical properties of the film, and geometry. The localized charge distribution produces an electric field that phase shifts subsequent electrons. As a result, each beam is rotated in the complex plane by the angle $\phi_{\mathrm{n}}$. Once the beams recombine in the image plane, phase contrast arises due to their interference, similar to the formation of lattice fringes in high-resolution transmission electron microscopy. b) The electron-optical set up for the diffraction mode allows to conjugate the image of the HFPP (i.e. diffraction pattern of the phase grating in it) with the detector. c) The image mode conjugates the object plane with the phase grating in it with the detector. 


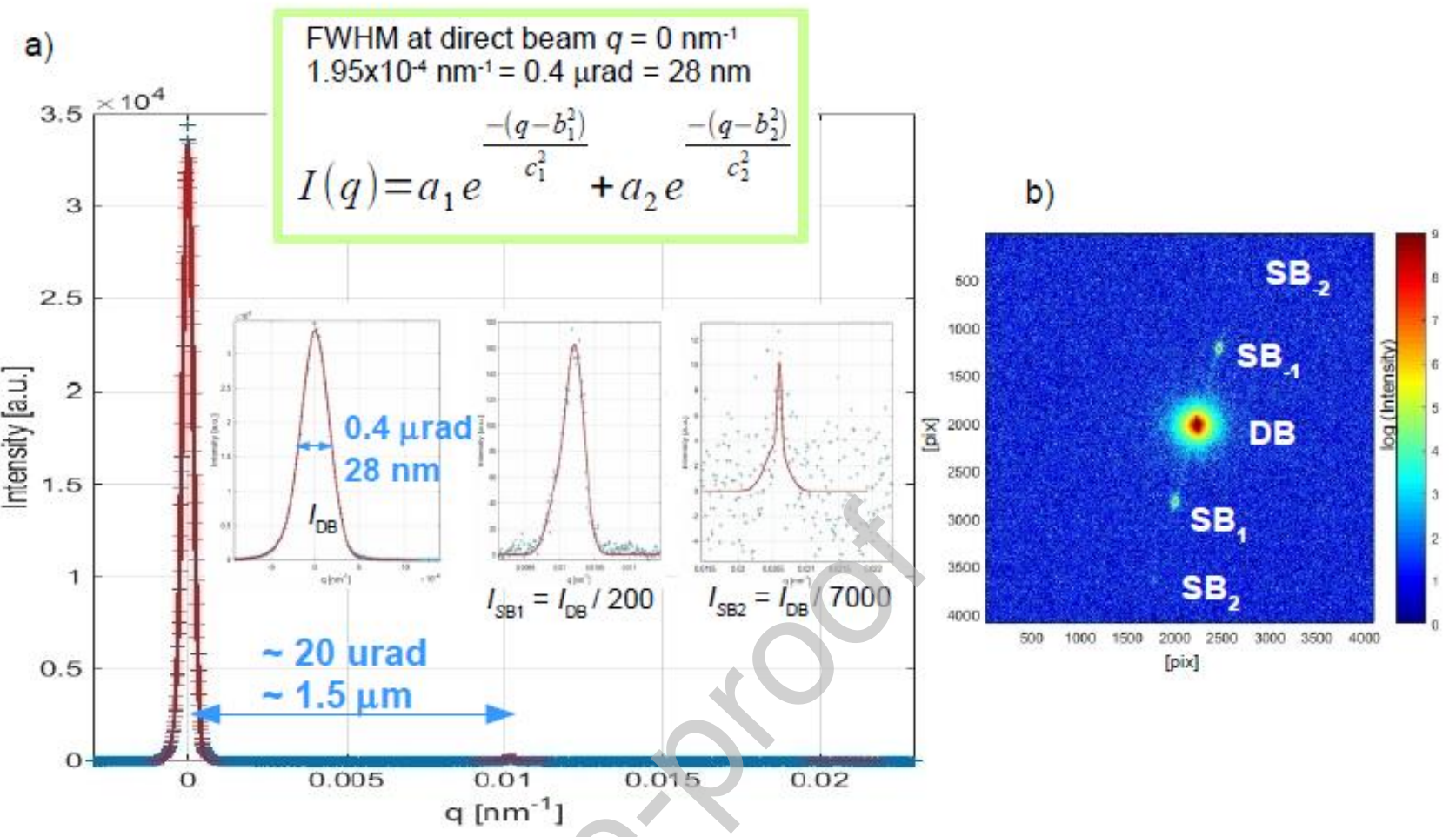

Figure 2

- Figure 2 (Color online) a) Beam current density at the back focal plane measured by recording the diffraction pattern of the phase grating. The fitted direct beam and the diffracted side beam intensity allows estimation the current density at the back focal plane of the objective lens where the HFPP is located. b) The acquired diffraction pattern, displayed as the log of the intensity. The line intensity profile for $\mathrm{DB}, 1^{\text {st }}$ order diffracted beam $\mathrm{SB}_{1}$, and $2^{\text {nd }}$ order diffracted beam, $\mathrm{SB}_{2}$, are shown together with the formula for a two-Gaussian curve used to fit the beam intensity profile. 

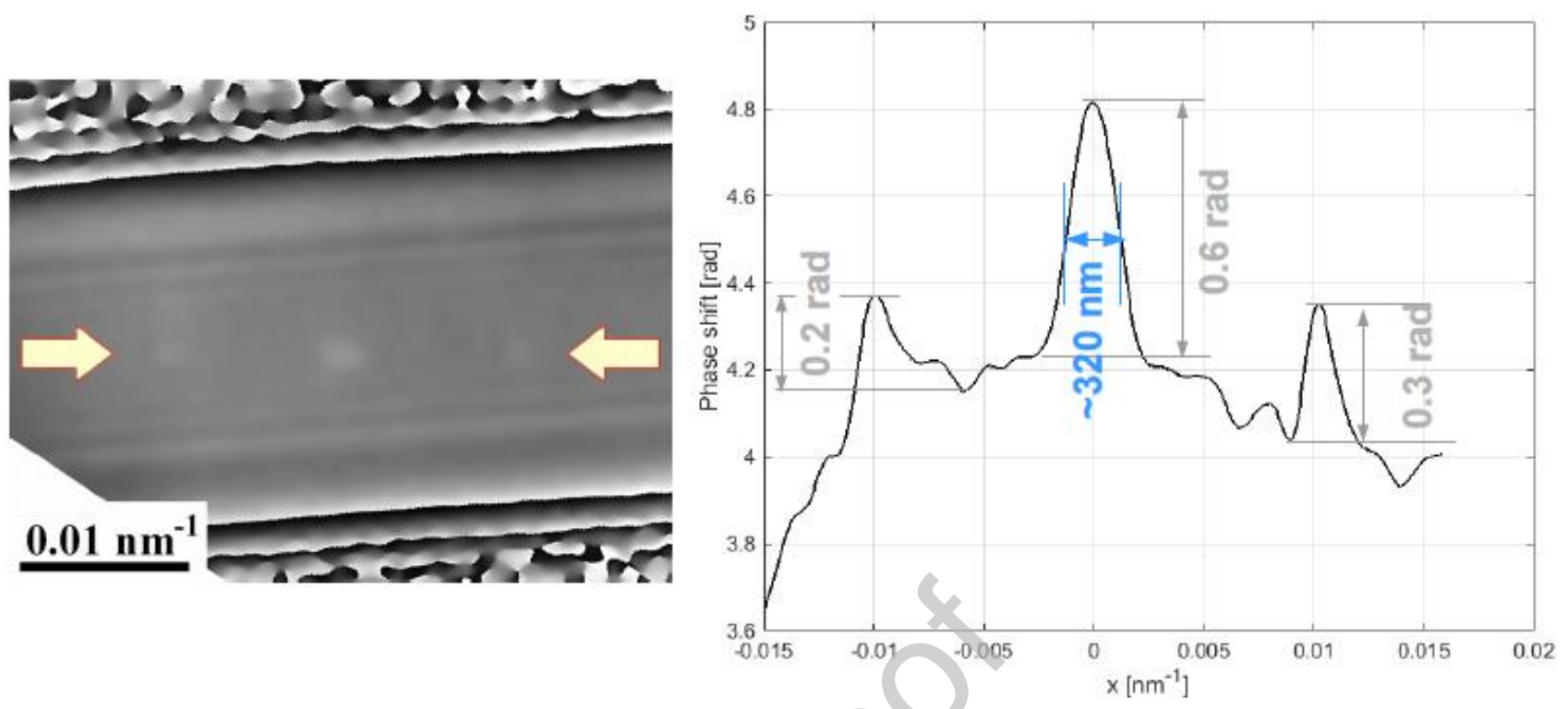

- Figure 3 (Color online) a) Phase shift after the end of the irradiation period as measured by electron holography. b) Phase shift profile extracted along the dashed line in a). The difference between the direct and diffracted beams is $\sim 0.3-0.4$ rad. The full width half maximum of the phase footprint measured by electron holography is $\sim 320 \mathrm{~nm}$. Note that both a) and b) depict the magnitude of the phase shift, the negative sign of the phase shift was omitted for clarity and easier visual comparison with Fig. 2. 
b)
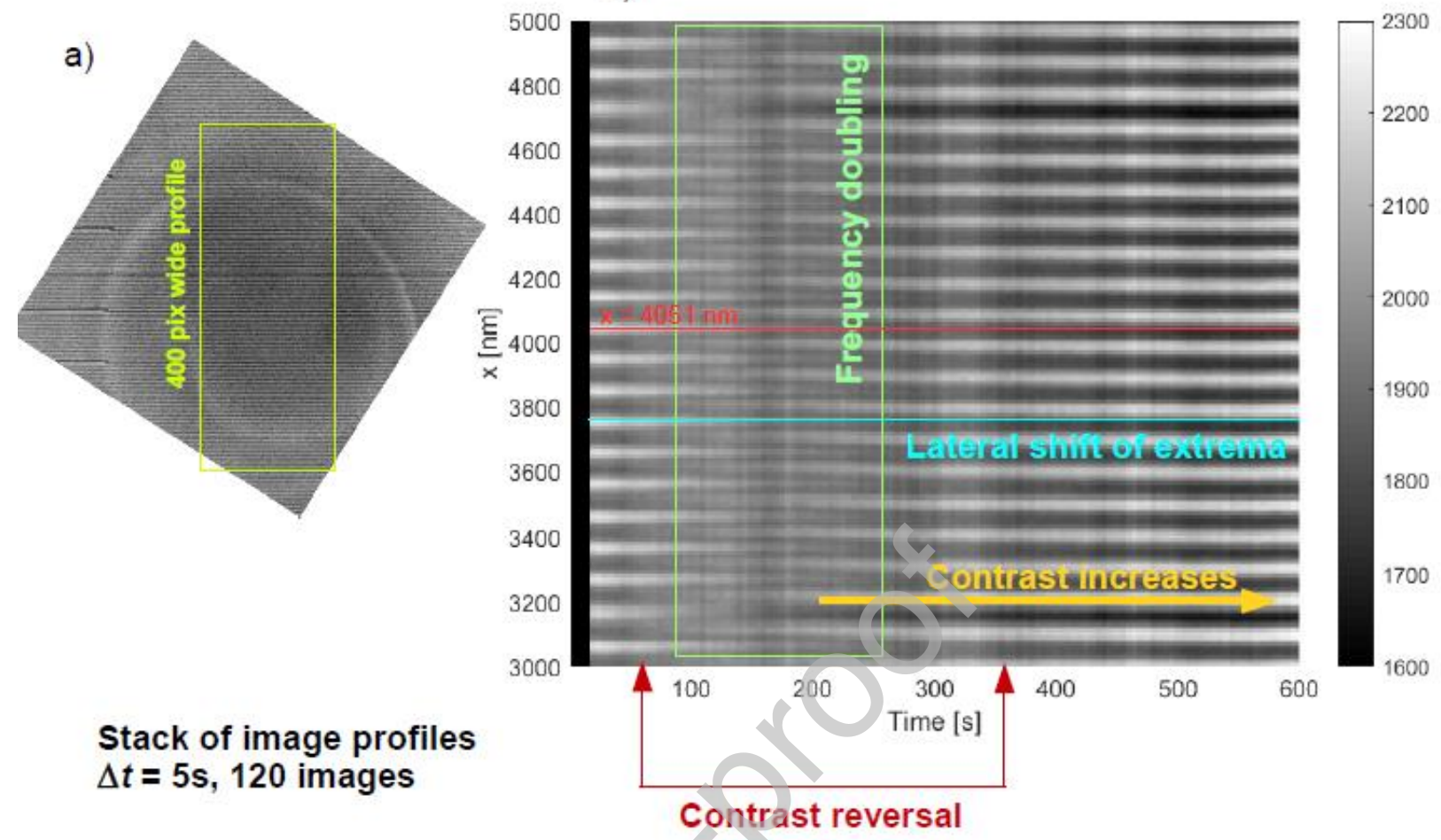

Stack of image profiles $\Delta t=5 \mathrm{~s}, 120$ images

\section{Contrast reversal}

- Figure 4 (Color online) Example evolution of the image intensity of the phase grating. a) HFPP Image extracted from a stack of 120 images, collected at 5 s intervals. A 400 pixel wide profile indicating the area summed to provide individual intensity profiles, stacked in a time series shown in b). The region of frequency doubling, contrast reversal, lateral shift of fringes, and continuing contrast increase with time, are indicated in b).

a)

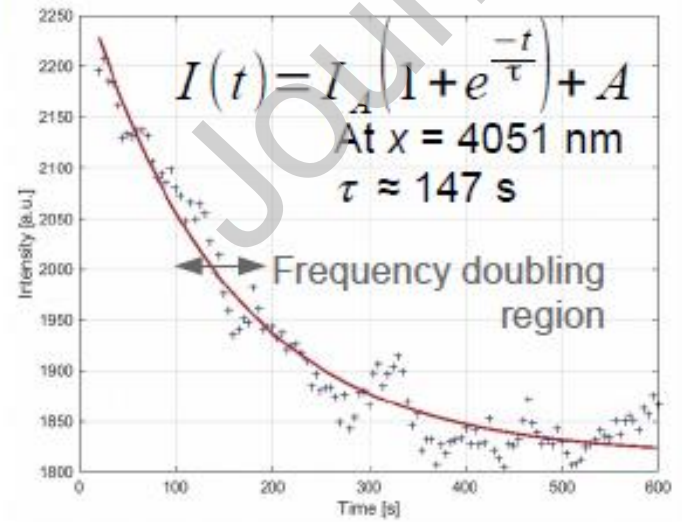

b)

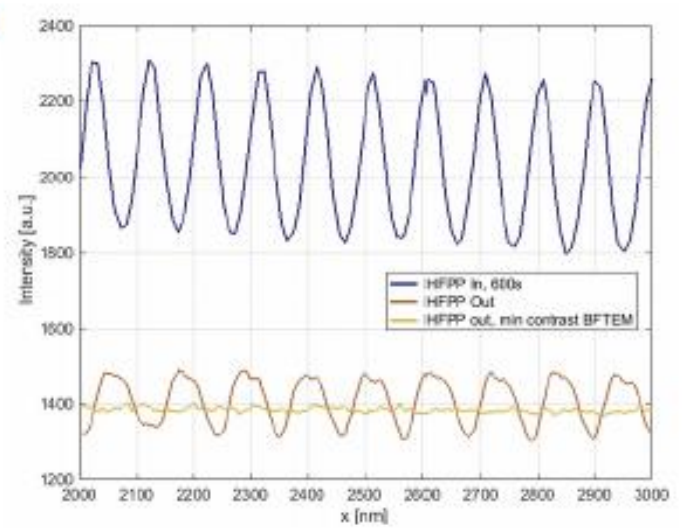

- Figure 5 (Color online) a) Settling time related to difference $\phi_{\mathrm{DB}}-\phi_{\mathrm{SB}}$. Profile extracted along red line at $x=4051 \mathrm{~nm}$ in Figure 4. The approximate region where frequency doubling occurs is marked with a grey arrow. b) Image intensity profile for HFPP (top, blue), BFTEM with HFPP 
retracted from the beam but no focus adjustment (red, sinusoidal-like line) and BFTEM with focus adjusted for minimum contrast (yellow, nearly flat line). The BFTEM profiles were offset with respect to HFPP profile.
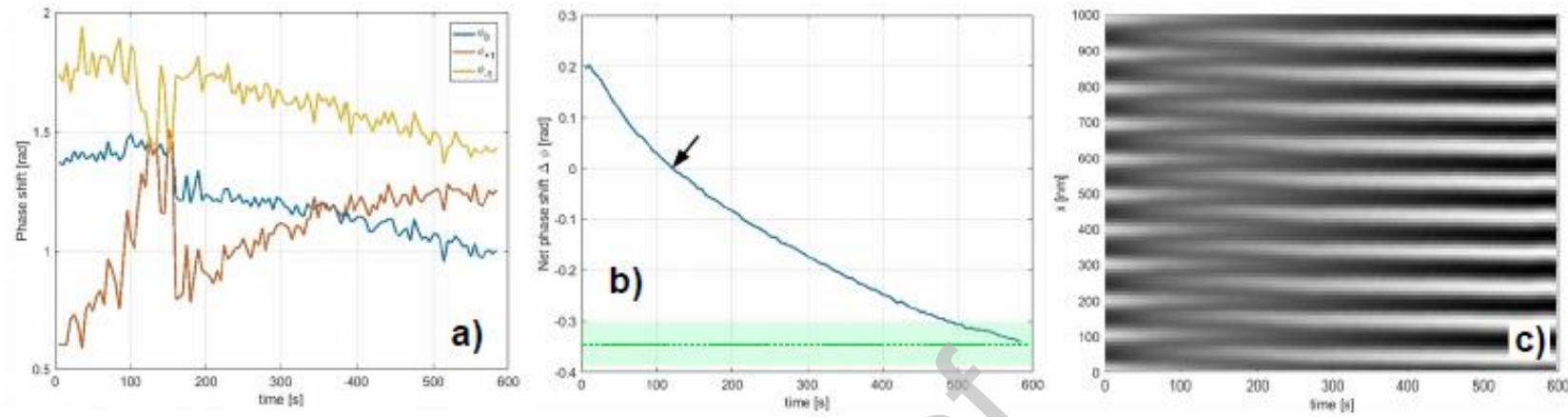

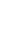

- Figure 6 (Color online) a) Phase shift of the direct and two first order diffracted beams extracted by fitting Eq. 5 to the experimental data shown in Figure 4. b) Net phase shift $\Delta \phi=\phi_{0}-1 / 2\left(\phi_{+1}+\phi_{\text {- }}\right.$ 1). The green dashed line with shaded region corresponds to the phase shift difference measured by off axis electron holography after the experiment (see Figure 3). c) An intensity profile simulated using the phase shift extracted in a) and b). The intensity profile stack in c) reproduces the features observed in the experimental data shown in Figure 4b. 

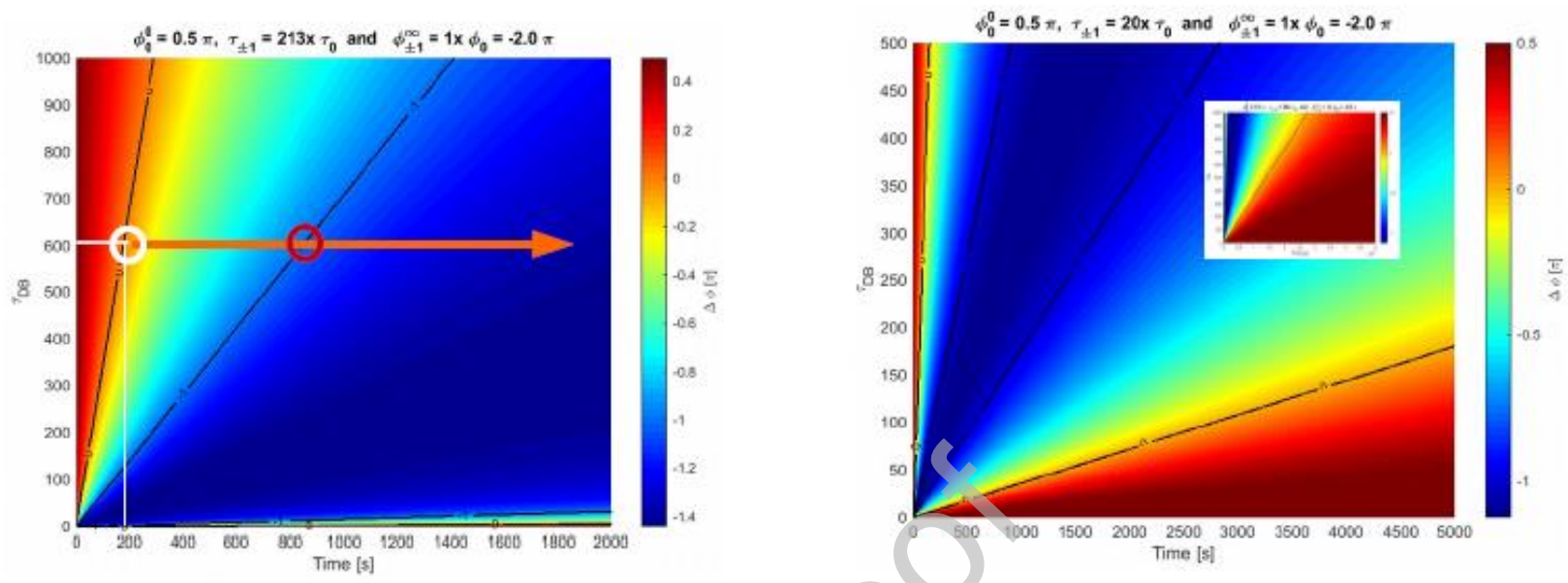

- Figure 7 (Color online) Conditions illustrating the image frequency doubling and HFPP settling rate that takes place at net phase shift $\Delta \phi=n \pi$ with $n$ integer. a) The settling time constant for the side beams phase shift is $213 \mathrm{x}$ larger than that of the direct beam phase shift. $\mathrm{b}$ ) The settling time constant for the side beams phase shift is now 20x larger than that of the direct beam phase shift. The inset covers the whole experiment duration, $\sim 14 \mathrm{hrs}$. The initial phase shift, $\pi / 2$, and the asymptotic phase shift, $-2 \pi$, are the same in both cases. The condition for frequency doubling is marked by a black line.

- Conflict of Interest

- We declare, that according to our best knowledge, we have no competing interests. 